\title{
The eleven-year switch of peptide aptamers Pierre Colas
}

Address: Station Biologique, CNRS, UPS 2682, Place Georges Teissier, 29280 Roscoff, France. Email: colas@sb-roscoff.fr

Published: 31 January 2008

Journal of Biology 2008, 7:2 (doi:10.1 186/jbiol64)

The electronic version of this article is the complete one and can be found online at http://jbiol.com/content/7/I/2

(C) 2008 BioMed Central Ltd

\begin{abstract}
Peptide aptamers are combinatorial recognition proteins that were introduced more than ten years ago. They have since found many applications in fundamental and therapeutic research, including their recent use in microarrays to detect individual proteins from complex mixtures.
\end{abstract}

Peptide aptamers are artificial recognition molecules that consist of a variable peptide sequence inserted into a constant scaffold protein [1]. The features that distinguish peptide aptamers from other classes of constrained combinatorial proteins (such as antibodies, antibody fragments and other non-antibody scaffold-based molecules) include their small size, their simple design and their disulfideindependent folding; the latter enables them to function inside living cells, unlike antibodies (Figure 1).

Peptide aptamers have been selected, using yeast two-hybrid methods, to bind to a wide range of cellular, viral and bacterial target proteins involved in a variety of regulatory pathways [2]. In most cases they have a high binding specificity, enabling them to discriminate between different closely related proteins within a functional family, or even between different allelic variants of a given protein [3]. In all cases, some of the aptamers tested have been shown to inhibit the function of their cognate targets and to cause phenotypes in the experimental models in which they were expressed. The use of peptide aptamers has thus enabled the dissection of molecular regulatory pathways by specifically probing protein functions, or sometimes even protein interactions.

The excellent recognition specificity and high binding affinity typical of peptide aptamers have suggested that they could be used in the many protein detection methods for which antibodies are currently used. The work by Wälti and colleagues published in this issue of Journal of Biology [4] turns this possibility into reality, by establishing that peptide aptamers can be immobilized on microarrays, which can then be used to detect and quantify proteins from complex solutions.

At least three important challenges must be overcome in order to generate microarrays that enable protein analysis at a proteomic scale. Wälti and colleagues [4] offer convincing solutions to each of them. A first challenge is to obtain collections of binding reagents that can specifically recognize proteins (and ideally the many isoforms generated by differential splicing and post-translational modifications) and also whose properties, such as stability and target binding, are homogeneous when arrayed on solid surfaces. In contrast to antibodies, which tend to be fragile, the simple design of peptide aptamers confers a greater robustness and probably enhances long-term stability. Moreover, peptide aptamers have relatively homogeneous target binding affinities [5], which is useful in protein detection as comparable protein levels generate comparable detection signals. The authors [4] used a new aptamer scaffold (STM, derived from stefin $A$, an intracellular inhibitor of cathepsins) instead of thioredoxin A, which has been the scaffold used most in other peptide aptamer applications. The STM scaffold has been engineered to abolish all its interactions with human proteins [6]; this feature should provide a better signal-to-noise ratio in protein detection. 


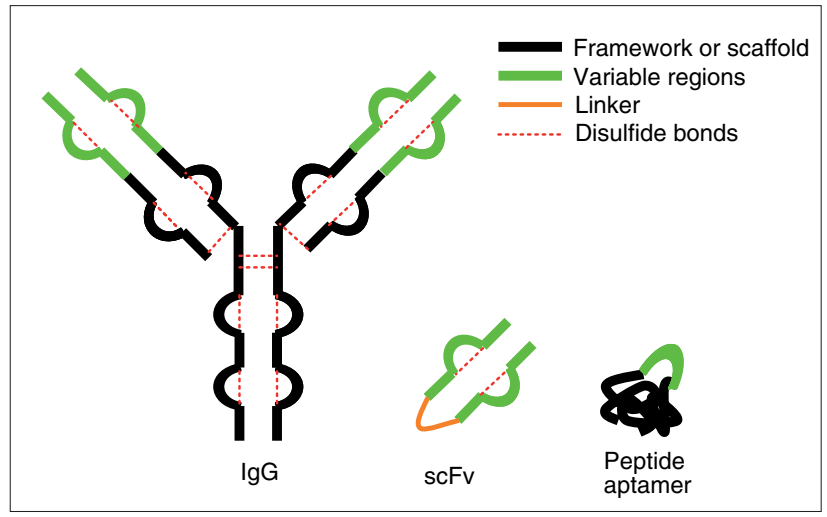

Figure I

Comparison between different constrained combinatorial recognition proteins, showing their approximate size and complexity (not to scale). IgG, immunoglobulin G; scFv, single-chain Fv antibody fragment.

A second challenge is to produce high-density arrays without compromising the sensitivity and specificity of protein detection. Wälti and colleagues [4] used a masking/unmasking procedure in which closely spaced gold electrodes were 'functionalized' by the attachment of specific aptamers; the electrodes were more than an order of magnitude smaller than the feature size currently used in protein arrays. These arrays are produced using conventional silicon manufacturing technology, which means that aptamer arraying could reach the nanometer scale in the future.

A third challenge is to develop very sensitive protein detection methods that do not compromise the structures of the proteins to be detected and that allow high-density multiplex binding measurements. The authors [4] opted for a label-free, electrochemical method that monitors local variations in the impedance of the electrochemical layer above the surface of the gold electrode. Capture of protein molecules by an aptamer-functionalized electrode perturbs the electrical properties of the layer and thus its impedance, as measured by applying an electrochemical potential to the electrode (Figure 2).

Using this method, Wälti and colleagues [4] show that an array of ten peptide aptamers can detect target proteins from complex mixtures, at a sensitivity that is relevant to that required to study clinical samples, and with a linear dynamic range that covers more than three orders of magnitude.

This work opens the way to an important extension of the therapeutic research applications of peptide aptamers, which are already used throughout the multi-step process of drug discovery (Figure 3) [1]. The inadequate validation of therapeutic targets is widely recognized as a major cause of high attrition rates and productivity decrease in drug discovery. The ability of peptide aptamers to selectively target and modulate the function of intracellular proteins makes them valuable tools for target validation, as they introduce perturbations that differ from those caused by gene knockout or knockdown methods [7] and that are arguably much more similar to perturbations caused by a small-molecule drug.

The mode of action of bioactive peptide aptamers has been explored in several studies. In most cases, aptamers have been shown to inhibit protein-protein interactions [3,7-9]. Some aptamers are selected for their ability to bind to transcription factors and have also been shown to inhibit protein-DNA interactions, either by masking the DNAbinding domain [10] or by inhibiting a protein interaction required for DNA binding $[10,11]$. Recently, however, an alternative mode of inhibition has been described for peptide aptamers selected against the hepatitis B virus core protein and the human papillomavirus E6 oncoprotein [12]. In both cases, fusion proteins comprising a histidine tag, an aptamer and the herpes simplex virus VP22 protein (which has been shown to improve gene transfer by spreading from a primary transfected cell to surrounding cells) were found to sequester their cognate targets into perinuclear inclusion bodies called aggresomes, thus depleting the pool of soluble target proteins from the cells. This sequestration phenomenon will probably be observed less frequently when 'naked' peptide aptamers are expressed. Finally, some peptide aptamers have been shown to activate rather than inhibit the function of their cognate target proteins, thus confirming that aptamer-induced perturbations are more similar to those caused by small molecules than to those caused by reverse genetics methods $[13,14]$.

Beyond the validation of target candidates, phenotypic selection of peptide aptamers can be a powerful approach for the identification of new therapeutic targets. Peptide aptamers have been isolated for their ability to confer phenotypes on yeast [15-17], bacteria [18] and human [14] cells. Some of these isolated aptamers have been used as baits to perform yeast two-hybrid screening of genomic or cDNA libraries and to identify their cognate targets. These interrogations of biological pathways have revealed new functions for open reading frames and proteins or have linked them to pathways in which they were previously not known to be involved.

Peptide aptamers can also have a direct impact on the discovery of new therapeutic molecules in two different ways. First, it is conceivable that peptide aptamers could themselves be used as biotherapeutics. Peptide aptamers selected for their ability to bind to the intracellular domain 


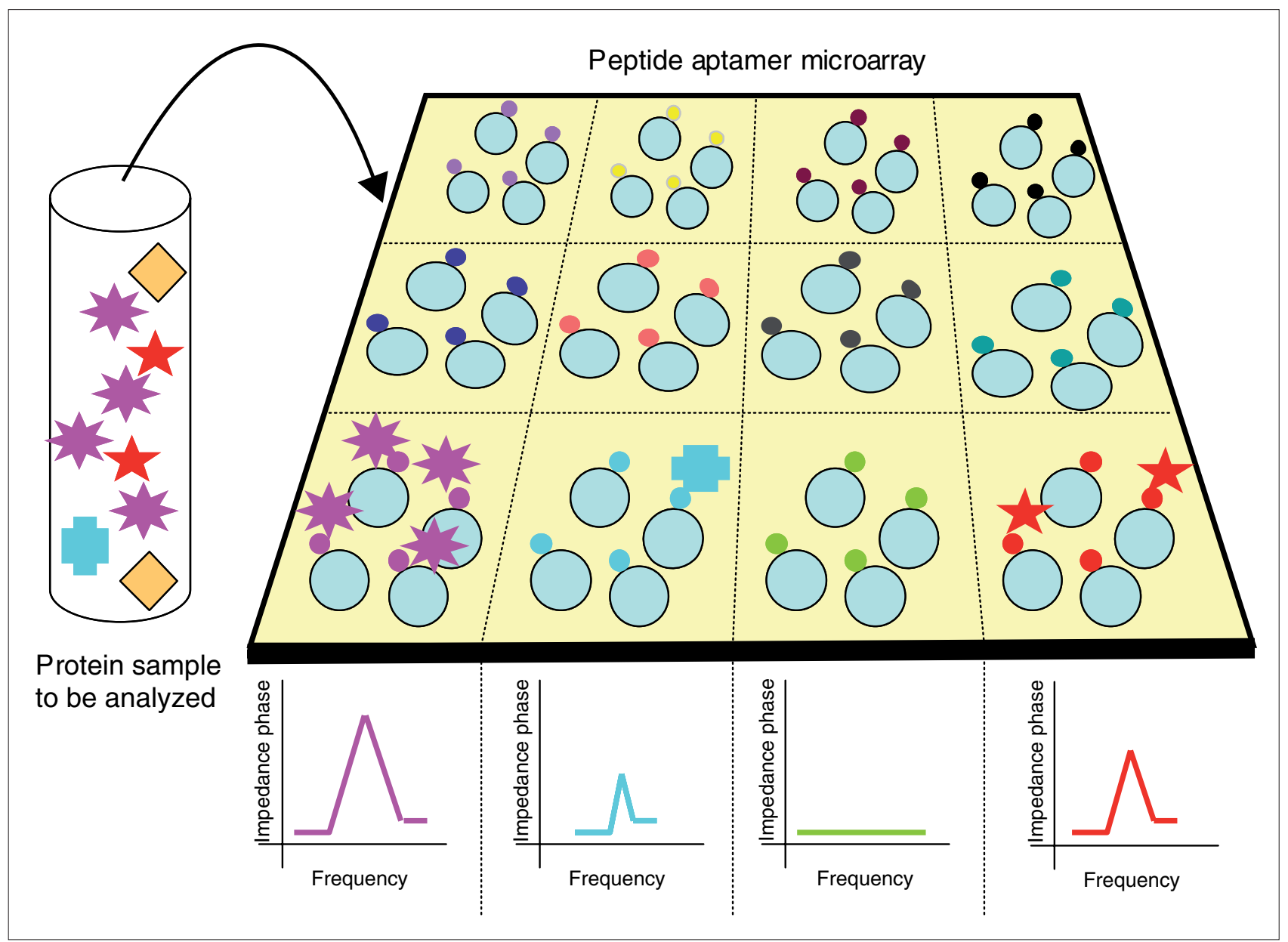

Figure 2

An electrochemical protein detection method using a peptide aptamer microarray [4]. A sample containing five different proteins is shown on the left, and an array of 12 different peptide aptamers is shown on the right (not to scale). Large gray spheres represent the aptamer scaffold protein; small colored spheres represent the aptamer variable regions, which confer binding specificity to cognate target proteins; and large colored shapes represent the cognate target proteins of the aptamers with matching colors. Protein detection is illustrated only for the first row of peptide aptamers. The binding of a protein to its cognate peptide aptamer perturbs the electrochemical properties of the layer above the electrode, which alters the impedance and its phase, as measured by applying an electrical signal of varying frequency to each electrode. An alteration in impedance is proportional to the amount of captured protein.

of the epidermal growth factor receptor and to the transcription factor Stat3 were fused to a polyarginine protein transduction domain (PTD), produced in Escherichia coli, and shown to inhibit the growth of tumor cells in vitro $[10,19]$. More recently, peptide aptamers directed against the prion-related protein $\operatorname{Prp}^{\mathrm{C}}$ were produced in E. coli without a PTD, added to prion-infected neuroblastoma cells, and shown to reduce formation of the pathogenic prion isoform $\operatorname{Prp}^{S c}$ [20]. Lengthy preclinical studies will be needed to determine whether peptide aptamers are likely to become biotherapeutic molecules, but this exciting prospect is worth the effort.
Peptide aptamers are also used to guide the discovery of small-molecule drugs targeting specific, alternative molecular surfaces on protein targets. A straightforward approach aimed at mapping aptamer-binding sites on target proteins has been developed [1]. When available, this structural information brings a whole new dimension to target validation, because what is validated is a specific molecular surface on a target protein [1]. When this surface is deemed 'druggable' (accessible to drug-like small molecules), the cognate peptide aptamer can be used like a 'cross-hair' in a high-throughput screening assay to identify small molecules that disrupt the target-aptamer interaction. This displace- 


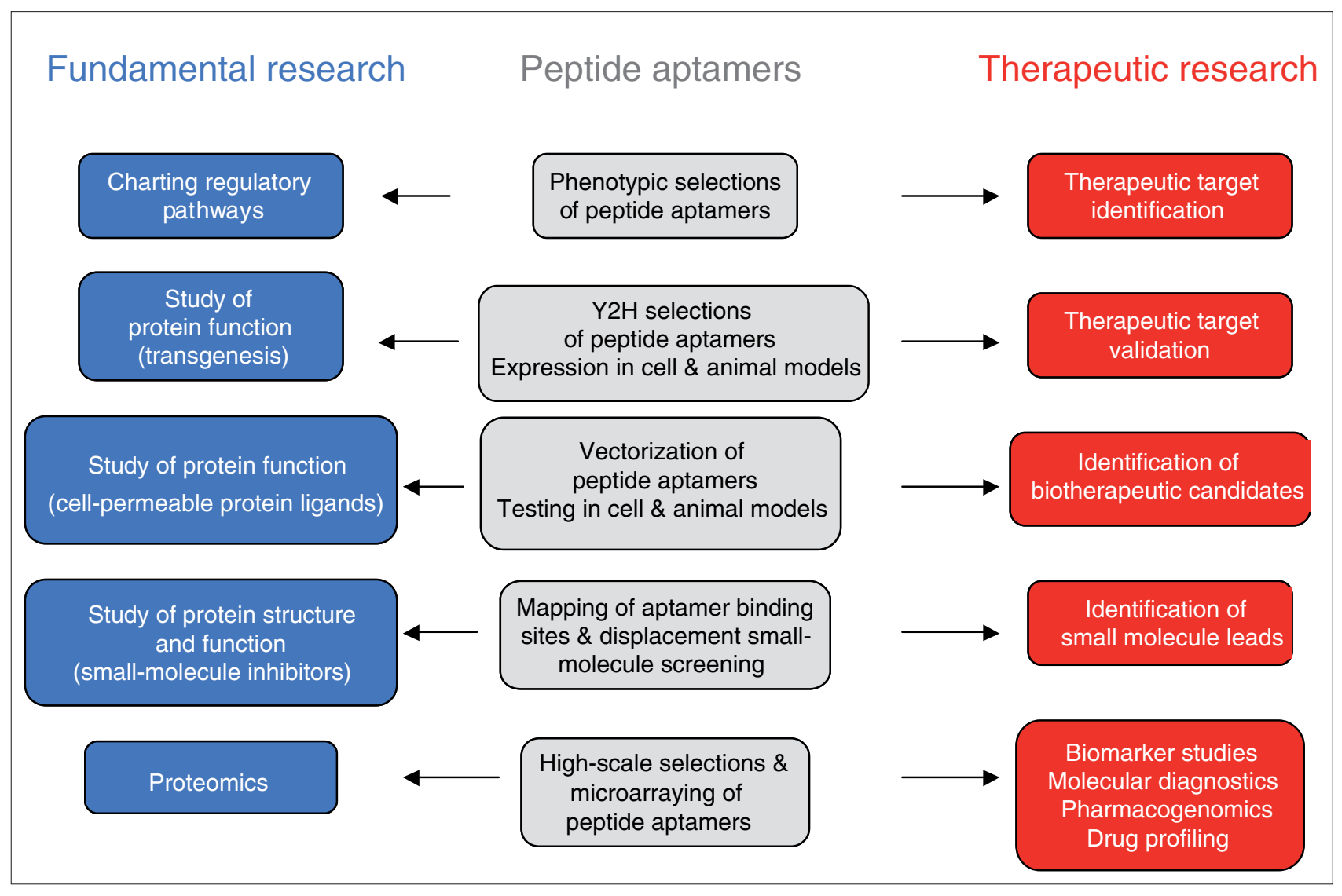

Figure 3

Fundamental and therapeutic research applications of peptide aptamers. The advances shown in the middle column can be used for the applications shown in fundamental (left) and therapeutic (right) research. $\mathrm{Y} 2 \mathrm{H}$, yeast two-hybrid.

ment assay rests on the premise that a peptide aptamer and a small molecule that bind to the same molecular surface on a target protein probably trigger the same biological effects. A duplex high-throughput yeast two-hybrid assay has been developed and is now being used against various target proteins $[1,21]$.

Analytical protein microarrays will probably have a significant impact on many areas of fundamental and therapeutic research [22] (Figure 3). The robustness and the homogeneous behavior of peptide aptamers conferred by their simple design should greatly facilitate the development and use of peptide-aptamer-based microarrays, which will offer a valuable alternative to antibody and antibody-fragment microarrays. However, two important problems will need to be addressed to scale peptide-aptamer-based microarrays up to a level at which they will deliver all their benefits. First, the coupling method used by Wälti and colleagues [4] must be improved in order to produce high-complexity arrays rapidly. Second, high-throughput peptide aptamer selection must be made possible. Recent improvements in the yeast two-hybrid selection of peptide aptamers [23] combined with the use of automated procedures [24] will help achieve a higher selection throughput, but the use of in vitro selection methods such as the bacterial flagellum display system [25] will probably be required to assemble proteome-scale collections of peptide aptamers.

Astronomers teach us that one of the most conspicuous phenomena on the surface of the sun is the appearance and disappearance of dark, irregularly shaped areas caused by violent eruptions; these are known as sunspots. Although sunspots can occur unexpectedly, a regular cycle of sunspot activity has been observed, with both a minimum and maximum level occurring approximately every 11 years. Around 11 years ago, a significant eruption occurred in the field of combinatorial biology with the first publication describing the selection of peptide aptamers by a yeast twohybrid method [5]. Over the past ten years, as the technology has matured and found various applications, a number 
of subsequent eruptions have occurred. A recent series of exciting reports exploring modes of action of peptide aptamers $[7,12,14,20]$ and describing new technology developments and applications $[1,4,21,23]$ marks an intense period of activity. With the latest remarkable eruption reported in this issue of Journal of Biology [4], we are now undoubtedly witnessing the 11-year switch of peptide aptamers.

\section{References}

I. Baines IC, Colas P: Peptide aptamers as guides for small molecule drug discovery. Drug Discov Today 2006, I I:334-34 I.

2. Hoppe-Seyler F, Crnkovic-Mertens I, Tomai E, Butz K: Peptide aptamers: specific inhibitors of protein function. Curr Mol Med 2004, 4:529-538.

3. Xu CW, Luo Z: Inactivation of Ras function by allele-specific peptide aptamers. Oncogene 2002, 21:5753-5757.

4. Evans D, Johnson S, Laurenson S, Giles Davies A, Ko Ferrigno P, Wälti C: Electrical protein detection in cell lysates using highdensity peptide aptamer microarrays. J Biol 2008, 7:3.

5. Colas P, Cohen B, Jessen T, Grishina I, McCoy J, Brent R: Genetic selection of peptide aptamers that recognize and inhibit cyclindependent kinase 2. Nature 1996, 380:548-550.

6. Woodman R, Yeh JT, Laurenson S, Ferrigno PK: Design and validation of a neutral protein scaffold for the presentation of peptide aptamers. J Mol Biol 2005, 352: I I I 8- I I 33.

7. Abed N, Bickle M, Mari B, Schapira M, Sanjuan-España R, Robbe Sermesant K, Moncorgé O, Mouradian-Garcia S, Barbry P, Rudkin $\mathrm{BB}$, Fauvarque MO, Michaud-Soret I, Colas P: A comparative analysis of perturbations caused by a gene knockout, a dominant negative allele, and a set of peptide aptamers. Mol Cell Proteomics 2007, 6:21 10-2I 21 .

8. Cohen BA, Colas P, Brent R: An artificial cell-cycle inhibitor isolated from a combinatorial library. Proc Natl Acad Sci USA 1998, 95: | $4272-14277$.

9. Geyer CR, Colman-Lerner A, Brent R: "Mutagenesis" by peptide aptamers identifies genetic network members and pathway connections. Proc Natl Acad Sci USA 1999, 96:8567-8572.

10. Nagel-Wolfrum K, Buerger C, Wittig I, Butz K, Hoppe-Seyler F, Groner B: The interaction of specific peptide aptamers with the DNA binding domain and the dimerization domain of the transcription factor Stat 3 inhibits transactivation and induces apoptosis in tumor cells. Mol Cancer Res 2004, 2: I70- I82.

I I. Fabbrizio E, Le Cam L, Polanowska J, Kaczorek M, Lamb N, Brent R, Sardet C: Inhibition of mammalian cell proliferation by genetically selected peptide aptamers that functionally antagonize E2F activity. Oncogene 1999, 18:4357-4363.

12. Tomai E, Butz K, Lohrey C, von Weizsacker F, Zentgraf H, HoppeSeyler F: Peptide aptamer-mediated inhibition of target proteins by sequestration into aggresomes. J Biol Chem 2006, 28I:2 I345-2I 352.

13. Nouvion AL, Thibaut J, Lohez OD, Venet S, Colas P, Gillet G, Lalle P: Modulation of $\mathrm{Nr}-\mathrm{I} 3$ antideath activity by peptide aptamers. Oncogene 2006, 26:701-710.

14. de Chassey B, Mikaelian I, Mathieu AL, Bickle M, Olivier D, Nègre $D$, Cosset FL, Rudkin BB, Colas P: An antiproliferative genetic screening identifies a peptide aptamer that targets calcineurin and up-regulates its activity. Mol Cell Proteomics 2007, 6:45 I-459.

I5. Caponigro G, Abedi MR, Hurlburt AP, Maxfield A, Judd W, Kamb A: Transdominant genetic analysis of a growth control pathway. Proc Natl Acad Sci USA 1998, 95:7508-75I3.

16. Norman TC, Smith DL, Sorger PK, Drees BL, O'Rourke SM, Hughes TR, Roberts CJ, Friend SH, Fields S, Murray AW: Genetic selection of peptide inhibitors of biological pathways. Science I999, 285:59|-595.

17. Geyer CR, Brent R: Selection of genetic agents from random peptide aptamer expression libraries. Methods Enzymol 2000, 328: $|7|-208$.
18. Blum JH, Dove SL, Hochschild A, Mekalanos J]: Isolation of peptide aptamers that inhibit intracellular processes. Proc Natl Acad Sci USA 2000, 97:224I-2246.

19. Buerger C, Nagel-Wolfrum K, Kunz C, Wittig I, Butz K, HoppeSeyler F, Groner B: Sequence-specific peptide aptamers, interacting with the intracellular domain of the epidermal growth factor receptor, interfere with Stat3 activation and inhibit the growth of tumor cells. J Biol Chem 2003, 278:376 I0-3762I.

20. Gilch S, Kehler C, Schatzl HM: Peptide aptamers expressed in the secretory pathway interfere with cellular PrPsc formation. $J \mathrm{Mol}$ Biol 2007, 371:362-373.

21. Bardou C, Borie C, Bickle M, Rudkin BB, Colas P: Peptide aptamers for small-molecule drug discovery. Methods $\mathrm{Mol} \mathrm{Biol}$ 2008, in press.

22. Borrebaeck CA, Wingren C: High-throughput proteomics using antibody microarrays: an update. Expert Rev Mol Diagn 2007, 7:673-686.

23. Bickle MBT, Dusserre E, Moncorgé O, Bottin H, Colas P: Selection and characterization of large collections of peptide aptamers through optimized yeast two-hybrid procedures. Nat Protoc 2006, I:|066-109|.

24. Albers M, Kranz H, Kober I, Kaiser C, Klink M, Suckow J, Kern R, Koegl M: Automated yeast two-hybrid screening for nuclear receptor-interacting proteins. Mol Cell Proteomics 2005, 4:205213.

25. Lu Z, Murray KS, Van Cleave V, LaVallie ER, Stahl ML, McCoy JM: Expression of thioredoxin random peptide libraries on the Escherichia coli cell surface as functional fusions to flagellin: a system designed for exploring protein-protein interactions. Biotechnology (NY) 1995, 13:366-372. 